\title{
Almost periodic solution for a delayed Lotka-Volterra system on time scales
}

Yongzhi Liao* and Lijun Xu

\section{"Correspondence:}

mathyzliao@163.com

School of Mathematics and

Computer Science, Panzhihua

University, Panzhihua, Sichuan 617000, China

\begin{abstract}
This paper is concerned with a delayed Lotka-Volterra system on time scales. By using the theory of exponential dichotomy on time scales and fixed point theory based on monotone operator, some simple conditions are obtained for the existence and uniqueness of positive almost periodic solution of the system. Further, by means of the theory of calculus on time scales and Lyapunov functional, the global attractivity of the almost periodic solution is also investigated. The main results in this paper improve and extend the previously known results. Finally, some examples are given to illustrate the feasibility and effectiveness of the main result.
\end{abstract}

MSC: 34K14; 34K20; 34N05; 92D25

Keywords: almost periodic solution; Lotka-Volterra; monotone operator; time scales

\section{Introduction}

Let

$$
f^{-}=\inf _{s \in \mathbb{T}} f(s), \quad f^{+}=\sup _{s \in \mathbb{T}} f(s), \quad m(f)=\lim _{T \rightarrow \infty} \frac{1}{T} \int_{0}^{T} f(s) \mathrm{d} s,
$$

where $f$ is a continuous bounded function defined on $\mathbb{T}, \mathbb{T}$ is a time scale.

The Lotka-Volterra type system with delay is very important in the models of multispecies population dynamics and has interesting applications in epidemiology, physics, chemistry, economics, biological science, and other areas (see [1-3]). The assumption of almost periodicity is a way of incorporating the time dependent variability of the environment, especially when the various components of the environment are periodic with not necessarily commensurate periods. Therefore, in recent years, more and more researchers have been studying the almost periodic solutions of the Lotka-Volterra system with delay [4-7].

In $[4,5]$, the authors considered the following Lotka-Volterra system with time delays:

$$
x_{i}^{\prime}(t)=x_{i}(t)\left[r_{i}(t)-a_{i i}(t) x_{i}(t)-\sum_{j=1, j \neq i}^{n} a_{i j}(t) x_{j}\left(t-\tau_{i j}(t)\right)\right], \quad i=1,2, \ldots, n,
$$

where all the coefficients of system (1.1) are positive continuous almost periodic functions. In [4], with the help of a variable substitution and by applying Schauder's fixed point theorem, sufficient conditions for the existence of positive almost periodic solutions are obtained.

@2014 Liao and Xu; licensee Springer. This is an Open Access article distributed under the terms of the Creative Commons Attribution License (http://creativecommons.org/licenses/by/2.0), which permits unrestricted use, distribution, and reproduction in any medium, provided the original work is properly cited. 
Theorem 1.1 ([4]) Assume that

$\left(\mathrm{N}_{1}\right) r_{i}^{-}>0, a_{i j}^{-}>0, i, j=1,2, \ldots, n$;

$\left(\mathrm{N}_{2}\right) \quad m\left[r_{i}(t)-\sum_{j=1, j \neq i}^{n} a_{i j}(t) G_{j}(t)\right]>0$, where

$$
G_{j}(t)=\left[\int_{-\infty}^{t} e^{-\int_{s}^{t} r_{j}(u) \mathrm{d} u} a_{i j}(s) \mathrm{d} s\right]^{-1}, \quad i, j=1,2, \ldots, n
$$

Then system (1.1) has at least one positive almost periodic solution.

In $[5,6]$, the authors studied the following Lotka-Volterra type system with continuously distributed delays:

$$
x_{i}^{\prime}(t)=x_{i}(t)\left[r_{i}(t)-a_{i i}(t) x_{i}(t)-\sum_{j=1, j \neq i}^{n} a_{i j}(t) x_{j}\left(t-\tau_{i j}(t)\right)-\sum_{j=1}^{n} \int_{-\infty}^{t} k_{i j}(t, \theta) x_{j}(\theta) \mathrm{d} \theta\right],
$$

where all the coefficients of system (1.2) are positive almost periodic functions. Applying Schauder's fixed point theorem, the theory of the comparison theorem and the Lyapunov functional, the following theorem can be obtained.

Theorem $1.2([5,6])$ Assume that $\left(\mathrm{N}_{1}\right)$ and the following conditions hold:

$\left(\mathrm{T}_{1}\right) \sup _{t \in \mathbb{R}} \tau_{i j}^{\prime}(t)<1, i, j=1,2, \ldots, n$;

$\left(\mathrm{T}_{2}\right) \inf _{t \in \mathbb{R}}\left[r_{i}(t)-\sum_{j=1, j \neq i}^{n} a_{i j}(t) M_{j}-\sum_{j=1}^{n} M_{j} \int_{-\infty}^{t} k_{i j}(t, \theta) \mathrm{d} \theta\right]>0$, where $M_{j}=\sup _{t \in \mathbb{R}} \frac{r_{j}(t)}{a_{j j}(t)}$, $i, j=1,2, \ldots, n$;

$\left(\mathrm{T}_{3}\right)$ There exists a constant $\rho>0$ such that

$$
\min _{1 \leq i \leq n} \inf _{t \in \mathbb{R}}\left[a_{i i}(t)-\sum_{j=1, j \neq i}^{n} \frac{a_{j i}\left(v_{j i}^{-1}(t)\right)}{1-\tau_{j i}^{\prime}\left(v_{j i}^{-1}(t)\right)}-\sum_{j=1}^{n} \int_{0}^{\infty} k_{j i}(t+\theta, t) \mathrm{d} \theta\right]>\rho,
$$

where $v_{j i}^{-1}(t)$ is the inverse function of $t-\tau_{i j}(t)$.

Then system (1.2) has a unique positive almost periodic solution which is globally attractive.

The aim of this paper is to use the fixed point theory based on monotone operator and the Lyapunov functional to investigate the positive almost periodic solution of LotkaVolterra type systems (1.1)-(1.2). The following useful results on systems (1.1)-(1.2) are obtained without condition $\left(\mathrm{N}_{2}\right)$ of Theorem 1.1 and condition $\left(\mathrm{T}_{2}\right)$ of Theorem 1.2.

Theorem 1.3 Assume that $\left(\mathrm{N}_{1}\right)$ holds, then system (1.1) has a unique positive almost periodic solution.

Theorem 1.4 Assume that $\left(\mathrm{N}_{1}\right),\left(\mathrm{T}_{1}\right)$, and $\left(\mathrm{T}_{3}\right)$ of Theorem 1.2 hold, then system (1.2) has a unique positive almost periodic solution which is globally attractive.

Many authors have argued that the discrete time models governed by difference equations are reflecting the reality in a better way than the continuous ones when the populations have nonoverlapping generations. Discrete time models can also provide efficient computational models of continuous models for numerical simulations (see [7-9]). For 
example, in [7], Li et al. studied an almost periodic solution of the following discrete LotkaVolterra system with delays:

$$
\left\{\begin{array}{l}
x_{1}(n+1)=x_{1}(n) \exp \left\{r_{1}(n)\left[1-\frac{x_{1}(n)}{K_{1}}-\mu_{2}(n) x_{2}\left(n-\tau_{1}\right)\right]\right\} \\
x_{2}(n+1)=x_{2}(n) \exp \left\{r_{2}(n)\left[1-\frac{x_{2}(n)}{K_{2}}-\mu_{1}(n) x_{1}\left(n-\tau_{2}\right)\right]\right\}
\end{array}\right.
$$

By means of the theory of comparison theorem and an almost periodic functional hull theory, the authors obtained the result that system (1.3) is persistent and has a unique positive almost periodic solution, which is globally attractive.

The study of dynamic equations on time scales goes back to its founder Stefan Hilger [10] and is a new area of still fairly theoretical exploration in mathematics. Motivating the subject is the notion that dynamic equations on time scales can build bridges between continuous and discrete equations. Further, the study of time scales has led to several important applications, for example, in the study of insect population models, neural networks, heat transfer, and epidemic models. Recently the topic on the almost periodic solutions of dynamic systems on time scales has been intensively investigated in many papers.

Motivated by the above statement, in this paper we will study the following LotkaVolterra system with delays on time scales:

$$
\begin{aligned}
x_{i}^{\Delta}(t)= & x_{i}(t)\left[r_{i}(t)-a_{i i}(t) x_{i}(t)-\sum_{j=1, j \neq i}^{n} a_{i j}(t) x_{j}^{\sigma}\left(t-\tau_{i j}(t)\right)\right. \\
& \left.-\sum_{j=1}^{n} \int_{-\infty}^{t} k_{i j}(t, \theta) x_{j}^{\sigma}(\theta) \Delta \theta\right],
\end{aligned}
$$

where $t \in \mathbb{T}$ is a periodic time scale, $0 \in \mathbb{T} ; r_{i}(t)>0, a_{i j}(t)>0$ and $\tau_{i j}(t)>0$ are all almost periodic functions, $k_{i j}(t, s)>0, \sup \left\{\int_{-\infty}^{t} k_{i j}(t, \theta) \Delta \theta, t \in \mathbb{T}\right\}<\infty, i, j=1,2, \ldots, n$. From the point of view of biology, we focus our discussion on the existence, uniqueness and stability of positive almost periodic solution of system (1.4) by using the theory of exponential dichotomy on time scales, fixed point theory based on monotone operator and Lyapunov functional.

The remainder of this paper is organized in the following way. In Section 2, we will introduce some necessary notations, definitions and lemmas which will be used to gain novel results. In Section 3, some conditions are derived ensuring the existence and uniqueness of positive almost periodic solution of system (1.4) by using the theory of exponential dichotomy on time scales and fixed point theorem of monotone operator. In Section 4, we establish sufficient conditions for the global attractivity of a unique almost periodic solution of system (1.1) and system (1.4) without delays by means of Lyapunov functional. The main result in Section 4 is illustrated by giving some examples in Section 5.

\section{Preliminaries}

Now, let us state the following definitions and lemmas, which will be useful in proving our main result.

Definition 2.1 ([11]) A time scale $\mathbb{T}$ is an arbitrary nonempty closed subset of the real set $\mathbb{R}$ with the topology and ordering inherited from $\mathbb{R}$. The forward jump operators $\sigma: \mathbb{T} \rightarrow \mathbb{T}$ 
and the graininess $\mu: \mathbb{T} \rightarrow \mathbb{R}^{+}$are defined, respectively, by

$$
\sigma(t):=\inf \{s \in \mathbb{T}: s>t\}, \quad \mu(t):=\sigma(t)-t .
$$

The point $t \in \mathbb{T}$ is called left-dense, left-scattered, right-dense or right-scattered if $\rho(t)=t$, $\rho(t)<t, \sigma(t)=t$ or $\sigma(t)>t$, respectively. Points that are right-dense and left-dense at the same time are called dense.

Definition 2.2 ([11]) A function $p: \mathbb{T} \rightarrow \mathbb{R}$ is said to be regressive provided $1+\mu(t) p(t) \neq 0$ for all $t \in \mathbb{T}^{k}$. The set of all regressive rd-continuous functions $f: \mathbb{T} \rightarrow \mathbb{R}$ is denoted by $\mathcal{R}$. Let $p \in \mathcal{R}$. The exponential function is defined by

$$
e_{p}(t, s)=\exp \left(\int_{s}^{t} \xi_{\mu(\tau)}(p(\tau)) \Delta \tau\right)
$$

where $\xi_{h(z)}$ is the so-called cylinder transformation:

$$
\xi_{h}(z)=\frac{1}{h} \log (1+z h), \quad h>0,
$$

where $\log$ is the principal logarithm function. For $h=0, \xi_{0}(z)=z$. The inverse transformation of the cylinder transformation $\xi_{h}$ is given by

$$
\xi_{h}^{-1}(z)=\frac{1}{h}\left(e^{z h}-1\right), \quad h>0 .
$$

When $h=0, \xi_{0}^{-1}(z)=z$.

Throughout this paper, we always make the following assumption for system (1.4):

$\left(\mathrm{H}_{1}\right) r_{i} \in \mathcal{R}, r_{i}^{-}>0$ and $\sum_{i=1}^{n}\left(a_{i j}^{-}+k_{i j}^{-}\right)>0$, where $k_{i j}^{-}:=\inf _{s \in \mathbb{R}} \int_{-\infty}^{s} k_{i j}(s, \theta) \Delta \theta, i, j=1,2, \ldots, n$.

Lemma 2.1 ([11]) Let $p, q \in \mathcal{R}$. Then

(i) $e_{0}(t, s) \equiv 1$ and $e_{p}(t, t) \equiv 1$;

(ii) $\frac{1}{e_{p}(t, s)}=e_{\ominus p}(t, s)$, where $\ominus p(t)=-\frac{p(t)}{1+\mu(t) p(t)}$;

(iii) $e_{p}(t, s) e_{p}(s, r)=e_{p}(t, r)$;

(iv) $e_{p}^{\Delta}(\cdot, s)=p e_{p}(\cdot, s)$.

Definition 2.3 ([11]) For $f: \mathbb{T} \rightarrow \mathbb{R}$ and $t \in \mathbb{T}^{k}$, the delta derivative of $f$ at $t$, denoted by $f^{\Delta}(t)$, is the number (provided it exists) with the property that given any $\epsilon>0$, there is a neighborhood $U \subset \mathbb{T}$ of $t$ such that

$$
\left|f(\sigma(t))-f(s)-f^{\Delta}(t)[\sigma(t)-s]\right| \leq \epsilon|\sigma(t)-s|, \quad \forall s \in U .
$$

Lemma 2.2 ([11]) Let $f, g$ be $\Delta$-differentiable functions on $\mathbb{T}^{k}$. Then

(i) $\left(k_{1} f+k_{2} g\right)^{\Delta}=k_{1} f^{\Delta}+k_{2} g^{\Delta}$ for any constants $k_{1}, k_{2}$;

(ii) $(f g)^{\Delta}(t)=f^{\Delta}(t) g(t)+f(\sigma(t)) g^{\Delta}(t)=f(t) g^{\Delta}(t)+f^{\Delta}(t) g(\sigma(t))$.

Lemma 2.3 ([11]) Assume that $g: \mathbb{R} \rightarrow \mathbb{R}$ is continuous, $g: \mathbb{T} \rightarrow \mathbb{R}$ is $\Delta$-differentiable on $\mathbb{T}^{\kappa}$, and $f: \mathbb{R} \rightarrow \mathbb{R}$ is continuously differentiable. Then there exists $c$ in the real interval 
$[t, \sigma(t)]$ with

$$
[f(g(t))]^{\Delta}=f^{\prime}(g(c)) g^{\Delta}(t)
$$

Lemma 2.4 ([11]) Assume that $p(t) \geq 0$ for $t \geq 0$. Then $e_{p}(t, s) \geq 1$.

Lemma 2.5 ([11]) Suppose that $p \in \mathcal{R}^{+}$. Then

(i) $e_{p}(t, s)>0$ for all $t, s \in \mathbb{T}$;

(ii) if $p(t) \leq q(t)$ for all $t \geq s, t, s \in \mathbb{T}$, then $e_{p}(t, s) \leq e_{q}(t, s)$ for all $t \geq s$.

Lemma 2.6 ([11]) Suppose that $p \in \mathcal{R}$ and $a, b, c \in \mathbb{T}$, then

$$
\left[e_{p}(c, \cdot)\right]^{\Delta}=-p\left[e_{p}(c, \cdot)\right]^{\sigma}, \quad \int_{a}^{b} p(t) e_{p}(c, \sigma(t)) \Delta t=e_{p}(c, a)-e_{p}(c, b) .
$$

Definition 2.4 ([12]) A time scale $\mathbb{T}$ is called a periodic time scale if

$$
\Pi:=\{\tau \in \mathbb{R}: t+\tau \in \mathbb{T}, \forall t \in \mathbb{T}\} \neq\{0\}
$$

Definition 2.5 ([13]) Let $\mathbb{T}$ be a periodic time scale. A function $x: \mathbb{T} \rightarrow \mathbb{R}^{n}$ is called almost periodic on $\mathbb{T}$, if for any $\epsilon>0$, the set

$$
E(\epsilon, x)=\{\tau \in \Pi:|x(t+\tau)-x(t)|<\epsilon, \forall t \in \mathbb{T}\}
$$

is relatively dense in $\mathbb{T}$; that is, there exists a constant $l=l(\epsilon)>0$, for any interval with length $l(\epsilon)$, there exists a number $\tau=\tau(\epsilon)$ in this interval such that

$$
\|x(t+\tau)-x(t)\|<\epsilon, \quad \forall t \in \mathbb{T}
$$

The set $E(\epsilon, x)$ is called the $\epsilon$-translation set of $x, \tau$ is called the $\epsilon$-translation number of $x$, and $l(\epsilon)$ is called the inclusion of $E(\epsilon, x)$.

Definition 2.6 ([14] $)$ Let $y \in C\left(\mathbb{T}, \mathbb{R}^{n}\right)$ and $P(t)$ be a $n \times n$ continuous matrix defined on $\mathbb{T}$. The linear system

$$
y^{\Delta}(t)=P(t) y(t), \quad t \in \mathbb{T}
$$

is said to be an exponential dichotomy on $\mathbb{T}$ if there exist constants $k, \lambda>0$, and a projection $S$ and the fundamental matrix $Y(t)$ satisfying

$$
\begin{aligned}
& \left\|Y(t) S Y^{-1}(s)\right\| \leq k e_{\ominus \lambda}(t, s), \quad \forall t \geq s, \\
& \left\|Y(t)(I-S) Y^{-1}(s)\right\| \leq k e_{\ominus \lambda}(s, t), \quad \forall t \leq s, t, s \in \mathbb{T} .
\end{aligned}
$$

Lemma 2.7 ([13]) If the linear system $y^{\Delta}(t)=P(t) y(t)$ has an exponential dichotomy, then the almost periodic system

$$
y^{\Delta}(t)=P(t) y(t)+g(t), \quad t \in \mathbb{T}
$$


has a unique almost periodic solution $y(t)$ which can be expressed as follows:

$$
y(t)=\int_{-\infty}^{t} Y(t) S Y^{-1}(\sigma(s)) g(s) \Delta s-\int_{t}^{\infty} Y(t)(I-S) Y^{-1}(\sigma(s)) g(s) \Delta s .
$$

Lemma 2.8 ([14]) If $P(t)=\left(a_{i j}(t)\right)_{n \times n}$ is a uniformly bounded $r d$-continuous matrix-valued function on $\mathbb{T}$, and there is a $\delta>0$ such that

$$
\left|a_{i i}(t)\right|-\sum_{j \neq i}\left|a_{i j}(t)\right|-\frac{1}{2} \mu(t)\left[\sum_{j \neq i}\left|a_{i j}(t)\right|\right]^{2}-\delta^{2} \mu(t) \geq 2 \delta, \quad t \in \mathbb{T}, i=1,2, \ldots, n,
$$

then $y^{\Delta}(t)=P(t) y(t)$ admits an exponential dichotomy on $\mathbb{T}$.

Lemma 2.9 ([10]) Suppose that $r: \mathbb{T} \rightarrow \mathbb{R}$ is regressive. Let $t_{0} \in \mathbb{T}$ and $y_{0} \in \mathbb{R}$. The unique solution of the initial value problem

$$
y^{\Delta}(t)=r(t) y(t)+g(t), \quad y\left(t_{0}\right)=y_{0}
$$

is given by

$$
y(t)=e_{r}\left(t, t_{0}\right) y_{0}+\int_{t_{0}}^{t} e_{r}(t, \sigma(s)) g(s) \Delta s
$$

Similar to the proofs of Lemmas $2.7-2.9$ which can be found in [13, 15-17], respectively, we have:

Lemma 2.10 Assume that $\left(\mathrm{H}_{1}\right)$ holds, then system (1.4) has a unique almost periodic solution $x=\left(x_{1}, x_{2}, \ldots, x_{n}\right)^{T}$ which can be expressed as follows:

$$
\begin{aligned}
x_{i}(t)= & \int_{t}^{+\infty} e_{r_{i}}(t, \sigma(s)) x_{i}(s)\left[a_{i i}(t) x_{i}(s)\right. \\
& \left.+\sum_{j=1, j \neq i}^{n} a_{i j}(s) x_{j}^{\sigma}\left(s-\tau_{i j}(s)\right)+\sum_{j=1}^{n} \int_{-\infty}^{s} k_{i j}(s, \theta) x_{j}^{\sigma}(\theta) \Delta \theta\right] \Delta s,
\end{aligned}
$$

where $i=1,2, \ldots, n$.

In order to obtain the existence and uniqueness of positive almost periodic solution of system (1.4), we first make the following preparations:

Let $E$ be a Banach space and $K$ be a cone in $E$. The semi-order induced by the cone $K$ is denoted by ' $\leq$ '. That is, $x \leq y$ if and only if $y-x \in K . x<y$ if $x \leq y$ and $x \neq y . x \gg y$ if $x-y \in \hat{K}$, where $\hat{K}$ is the interior of the cone $K$. A cone $K$ is called minihedral if for any pair $\{x, y\}, x, y \in E$, bounded above in order for there to exist a least upper bound $\sup \{x, y\}$. A cone $K$ is called normal if there exists a constant $N>0$ such that $x \leq y, x, y \in K$ implies $\|x\|_{E} \leq N\|y\|_{E}$.

Definition 2.7 ([18]) $\Phi: K \rightarrow K$ is said to be strongly superlinear, if for $\forall x>0$ and $t \in$ $(0,1)$, one has $\Phi(t x) \ll t \Phi x$. 
The following two lemmas cited from $[19,20]$ are useful for the proof of our main results in this section.

Lemma 2.11 ([21]) Let E be a real Banach space with an order cone $K$ satisfying

(a) $K$ has a nonempty interior,

(b) $K$ is normal and minihedral.

Assume that there are two points in $E, x_{*} \ll x^{*}$, and a monotone increasing compact continuous operator $\Phi:\left[x_{*}, x^{*}\right] \rightarrow$ E. If

$\Phi x_{*} \ll x_{*}, \quad x^{*} \ll \Phi x^{*}$,

then $\Phi$ has a fixed point $x \in\left[x_{*}, x^{*}\right]$. Here $\left[x_{*}, x^{*}\right]$ denotes the order interval $\left\{x \in E: x_{*} \leq\right.$ $\left.x \leq x^{*}\right\}$.

Lemma 2.12 ([22]) If $\Phi: K \rightarrow K$ is strongly superlinear and increasing, then $\Phi$ has at most one positive fixed point.

Consider the Banach space $E=A P\left(\mathbb{T}, \mathbb{R}^{n}\right)$ with the norm $\|x\|=\max _{1 \leq i \leq n}\left\{\left|x_{i}\right|_{0}\right\},\left|x_{i}\right|_{0}=$ $\sup _{t \in \mathbb{T}}\left|x_{i}(t)\right|$. Define the cone $K$ in $E$ by

$$
K=\left\{x=\left(x_{1}, x_{2}, \ldots, x_{n}\right)^{T} \in E: x_{i} \geq 0, i=1,2, \ldots, n\right\} .
$$

It is not difficult to verify that $K$ is normal, minihedral and has a nonempty interior.

Let the map $\Phi$ be defined by

$$
(\Phi x)(t)=\left(\left(\Phi_{1} x\right)(t),\left(\Phi_{2} x\right)(t), \ldots,\left(\Phi_{n} x\right)(t)\right)^{T},
$$

where

$$
\begin{aligned}
\left(\Phi_{i} x\right)(t)= & \int_{t}^{+\infty} e_{r_{i}}(t, \sigma(s)) x_{i}(s)\left[a_{i i}(t) x_{i}(s)\right. \\
& \left.+\sum_{j=1, j \neq i}^{n} a_{i j}(s) x_{j}^{\sigma}\left(s-\tau_{i j}(s)\right)+\sum_{j=1}^{n} \int_{-\infty}^{s} k_{i j}(s, \theta) x_{j}^{\sigma}(\theta) \Delta \theta\right] \Delta s,
\end{aligned}
$$

where $x \in K, t \in \mathbb{T}, i=1,2, \ldots, n$.

Let

$$
k_{i j}^{+}:=\sup _{s \in \mathbb{T}} \int_{-\infty}^{s} k_{i j}(s, \theta) \Delta \theta, \quad k_{i j}^{-}:=\inf _{s \in \mathbb{T}} \int_{-\infty}^{s} k_{i j}(s, \theta) \Delta \theta .
$$

By $\left(\mathrm{H}_{1}\right)$, one could choose some positive constants in $K, \underline{x}_{i}<\bar{x}_{i}$ satisfying

$$
\sum_{j=1}^{n}\left[a_{i j}^{+}+k_{i j}^{+}\right] \underline{x}_{j}<r_{i}^{-}, \quad \sum_{j=1}^{n}\left[a_{i j}^{-}+k_{i j}^{-}\right] \bar{x}_{j}>r_{i}^{+}, \quad i=1,2, \ldots, n .
$$

Lemma 2.13 $\Phi: D \rightarrow E$ is monotone increasing, where $D=\left[x_{*}, x^{*}\right], x_{*}=\left(\underline{x}_{1}, \underline{x}_{2}, \ldots, \underline{x}_{n}\right)^{T}$, $x^{*}=\left(\bar{x}_{1}, \bar{x}_{2}, \ldots, \bar{x}_{n}\right)^{T}$. 
Proof For $\forall x=\left(x_{1}, x_{2}, \ldots, x_{n}\right)^{T}, y=\left(y_{1}, y_{2}, \ldots, y_{n}\right)^{T} \in D, x \leq y$, i.e.,

$$
y-x \in K \quad \Rightarrow \quad x_{i} \leq y_{i}, \quad i=1,2, \ldots, n \text {. }
$$

So

$$
\begin{aligned}
& \left(\Phi_{i} x\right)(t)-\left(\Phi_{i} y\right)(t) \\
& =\int_{t}^{\infty} e_{r_{i}}(t, \sigma(s)) x_{i}(s)\left[a_{i i}(t) x_{i}(s)\right. \\
& \left.\quad+\sum_{j=1, j \neq i}^{n} a_{i j}(s) x_{j}^{\sigma}\left(s-\tau_{i j}(s)\right)+\sum_{j=1}^{n} \int_{-\infty}^{s} k_{i j}(s, \theta) x_{j}^{\sigma}(\theta) \Delta \theta\right] \Delta s \\
& \quad-\int_{t}^{\infty} e_{r_{i}}(t, \sigma(s)) y_{i}(s)\left[a_{i i}(t) y_{i}(s)\right. \\
& \left.\quad+\sum_{j=1, j \neq i}^{n} a_{i j}(s) y_{j}^{\sigma}\left(s-\tau_{i j}(s)\right)+\sum_{j=1}^{n} \int_{-\infty}^{s} k_{i j}(s, \theta) y_{j}^{\sigma}(\theta) \Delta \theta\right] \Delta s \\
& \leq 0,
\end{aligned}
$$

that is, $\left(\Phi_{i} x\right) \leq\left(\Phi_{i} y\right), i=1,2, \ldots, n$, which implies that $\Phi y-\Phi x \in K$. Then $\Phi x \leq \Phi y$. This completes the proof.

Lemma 2.14 $\Phi: D \rightarrow E$ is complete continuous.

Proof First, we show that $\Phi$ maps bounded sets into bounded sets. For $\forall x \in D$, we have

$$
\begin{aligned}
\left|\Phi_{i} x\right|_{0} & \leq \sum_{j=1}^{n}\left[a_{i j}^{+}+k_{i j}^{+}\right]\left|x_{i}\right|_{0}\left|x_{j}\right|_{0} \sup _{t \in \mathbb{T}} \int_{t}^{\infty} e_{r_{i}^{-}}(t, \sigma(s)) \Delta s \\
& =\frac{1}{r_{i}^{-}} \sum_{j=1}^{n}\left[a_{i j}^{+}+k_{i j}^{+}\right]\left|x_{i}\right|_{0}\left|x_{j}\right|_{0} \sup _{t \in \mathbb{T}} \int_{t}^{\infty}\left[-e_{r_{i}^{-}}(t, \sigma(s))\right]^{\Delta} \Delta s \\
& =\frac{1}{r_{i}^{-}} \sum_{j=1}^{n}\left[a_{i j}^{+}+k_{i j}^{+}\right]\left|x_{i}\right|_{0}\left|x_{j}\right|_{0} \sup _{t \in \mathbb{T}} e_{r_{i}^{-}}(t, \sigma(t)) \\
& \leq \frac{1}{r_{i}^{-}} \sum_{j=1}^{n}\left[a_{i j}^{+}+k_{i j}^{+}\right] \bar{x}_{i} \bar{x}_{j}, \quad i=1,2, \ldots, n .
\end{aligned}
$$

That is, $\Phi D$ is uniformly bounded. In addition, for $\forall t_{1}, t_{2} \in \mathbb{T}$ and $t_{1} \leq t_{2}$, notice that

$$
\begin{aligned}
& \left|\left(\Phi_{i} x\right)\left(t_{1}\right)-\left(\Phi_{i} x\right)\left(t_{2}\right)\right| \\
& =\mid \int_{t_{1}}^{\infty} e_{r_{i}}\left(t_{1}, \sigma(s)\right) x_{i}(s)\left[a_{i i}(s) x_{i}(s)\right. \\
& \left.\quad+\sum_{j=1, j \neq i}^{n} a_{i j}(s) x_{j}^{\sigma}\left(s-\tau_{i j}(s)\right)+\sum_{j=1}^{n} \int_{-\infty}^{s} k_{i j}(s, \theta) x_{j}^{\sigma}(\theta) \Delta \theta\right] \Delta s
\end{aligned}
$$




$$
\begin{aligned}
& \quad-\int_{t_{2}}^{\infty} e_{r_{i}}\left(t_{1}, \sigma(s)\right) x_{i}(s)\left[a_{i i}(s) x_{i}(s)\right. \\
& \left.+\sum_{j=1, j \neq i}^{n} a_{i j}(s) x_{j}^{\sigma}\left(s-\tau_{i j}(s)\right)+\sum_{j=1}^{n} \int_{-\infty}^{s} k_{i j}(s, \theta) x_{j}^{\sigma}(\theta) \Delta \theta\right] \Delta s \\
& +\int_{t_{2}}^{\infty} e_{r_{i}}\left(t_{1}, \sigma(s)\right) x_{i}(s)\left[a_{i i}(s) x_{i}(s)\right. \\
& \left.+\sum_{j=1, j \neq i}^{n} a_{i j}(s) x_{j}^{\sigma}\left(s-\tau_{i j}(s)\right)+\sum_{j=1}^{n} \int_{-\infty}^{s} k_{i j}(s, \theta) x_{j}^{\sigma}(\theta) \Delta \theta\right] \Delta s \\
& \quad-\int_{t_{2}}^{\infty} e_{r_{i}}\left(t_{2}, \sigma(s)\right) x_{i}(s)\left[a_{i i}(s) x_{i}(s)\right. \\
& \left.+\sum_{j=1, j \neq i}^{n} a_{i j}(s) x_{j}^{\sigma}\left(s-\tau_{i j}(s)\right)+\sum_{j=1}^{n} \int_{-\infty}^{s} k_{i j}(s, \theta) x_{j}^{\sigma}(\theta) \Delta \theta\right] \Delta s \mid \\
& \leq \mid \int_{t_{1}}^{t_{2}} e_{r_{i}}\left(t_{1}, \sigma(s)\right) x_{i}(s)\left[a_{i i}(s) x_{i}(s)\right. \\
& \left.\quad+\sum_{j=1, j \neq i}^{n} a_{i j}(s) x_{j}^{\sigma}\left(s-\tau_{i j}(s)\right)+\sum_{j=1}^{n} \int_{-\infty}^{s} k_{i j}(s, \theta) x_{j}^{\sigma}(\theta) \Delta \theta\right] \Delta s \mid \\
& \quad+\mid \int_{t_{2}}^{\infty}\left[e_{r_{i}}\left(t_{2}, \sigma(s)\right)-e_{r_{i}}\left(t_{1}, \sigma(s)\right)\right] x_{i}(s)\left[a_{i i}(s) x_{i}(s)\right. \\
& +\sum_{j=1}^{n}\left[a_{i j}^{+}+k_{i j}^{+} a_{i j}(s) x_{j}^{\sigma} \bar{x}_{j}\left|t_{2}-t_{1}\right|+\frac{1}{r_{i}^{-}} \sum_{j=1}^{n}\left[a_{i j}^{+}+k_{i j}^{+} \bar{x}_{i} \bar{x}_{j}\left|1-e_{r_{i}^{+}}\left(t_{1}, t_{2}\right)\right| \rightarrow 0, \quad a s t_{1} \rightarrow t_{2},\right.\right. \\
& \\
& \left.\quad s)+\sum_{j=1}^{n} \int_{-\infty}^{s} k_{i j}(s, \theta) x_{j}^{\sigma}(\theta) \Delta \theta\right] \Delta s \mid
\end{aligned}
$$

where $i=1,2, \ldots, n$. So $\Phi x$ is equicontinuous for any $x \in D=\left[x_{*}, x^{*}\right]$. Using the ArzelaAscoli theorem on time scales [23], $\Phi D$ is relatively compact. The Lebesgue's dominated convergence theorem on time scales [24] yields then that $\Phi$ is continuous. Hence, $\Phi$ is complete continuous. The proof of this lemma is complete.

\section{Almost periodic solutions}

In this section, we will utilize Lemmas 2.11 and 2.12 given in the previous section to establish some sufficient criteria for the existence and uniqueness of positive (almost) periodic solutions of system (1.4).

Theorem 3.1 Assume that $\left(\mathrm{H}_{1}\right)$ holds, then system (1.4) has a unique positive almost periodic solution.

Proof Now, we will use Lemma 2.11 to prove the existence of positive almost periodic solutions of system (1.4). By Lemmas 2.13 and 2.14, we know that $\Phi$ is a monotone increasing complete continuous operator on $D=\left[x_{*}, x^{*}\right]$. It remains to prove that

$$
\Phi x_{*} \ll x_{*}, \quad x^{*} \ll \Phi x^{*} .
$$


On the one hand, by the definition of $x_{*}$ it follows that

$$
\begin{aligned}
\Phi_{i} x_{*} & =\int_{t}^{\infty} e_{r_{i}}(t, \sigma(s)) \underline{x}_{i} \sum_{j=1}^{n}\left[a_{i j}(s)+\int_{-\infty}^{s} k_{i j}(s, \theta) \Delta \theta\right] \underline{x}_{j} \Delta s \\
& \leq \underline{x}_{i} \sum_{j=1}^{n}\left[a_{i j}^{+}+k_{i j}^{+}\right] \underline{x}_{j} \int_{t}^{\infty} e_{r_{i}}(t, \sigma(s)) \Delta s \\
& \leq \frac{1}{r_{i}^{-}} \underline{x}_{i} \sum_{j=1}^{n}\left[a_{i j}^{+}+k_{i j}^{+}\right] \underline{x}_{j} \\
& <\underline{x}_{i}, \quad i=1,2, \ldots, n,
\end{aligned}
$$

which implies that

$$
\Phi x_{*}=\left(\Phi_{1} x_{*}, \Phi_{2} x_{*}, \ldots, \Phi_{n} x_{*}\right)^{T}<\left(\underline{x}_{1}, \underline{x}_{2}, \ldots, \underline{x}_{n}\right)^{T}=x_{*} \Rightarrow \Phi x_{*} \ll x_{*} .
$$

On the other hand, one has from the definition of $x^{*}$ that

$$
\begin{aligned}
\Phi_{i} x^{*} & =\int_{t}^{\infty} e_{r_{i}}(t, \sigma(s)) \bar{x}_{i} \sum_{j=1}^{n}\left[a_{i j}(s)+\int_{-\infty}^{s} k_{i j}(s, \theta) \Delta \theta\right] \bar{x}_{j} \Delta s \\
& \geq \bar{x}_{i} \sum_{j=1}^{n}\left[a_{i j}^{-}+k_{i j}^{-}\right] \bar{x}_{j} \int_{t}^{\infty} e_{r_{i}}(t, \sigma(s)) \Delta s \\
& \geq \frac{1}{r_{i}^{+}} \bar{x}_{i} \sum_{j=1}^{n}\left[a_{i j}^{-}+k_{i j}^{-}\right] \bar{x}_{j} \\
& >\bar{x}_{i}, \quad i=1,2, \ldots, n,
\end{aligned}
$$

which implies that

$$
\Phi x^{*}=\left(\Phi_{1} x^{*}, \Phi_{2} x^{*}, \ldots, \Phi_{n} x^{*}\right)^{T}>\left(\bar{x}_{1}, \bar{x}_{2}, \ldots, \bar{x}_{n}\right)^{T}=x^{*} \Rightarrow \Phi x^{*} \gg x^{*} .
$$

Next, we prove the uniqueness of the positive almost periodic solution of system (1.4), using Lemma 2.12. For $x>0$ and $\lambda \in(0,1)$, it follows that

$$
\begin{aligned}
\Phi_{i}(\lambda x)= & \lambda^{2} \int_{t}^{\infty} e_{r_{i}}(t, \sigma(s)) x_{i}(t)\left[a_{i i}(t) x_{i}(s)\right. \\
& \left.+\sum_{j=1, j \neq i}^{n} a_{i j}(s) x_{j}^{\sigma}\left(s-\tau_{i j}(s)\right)+\sum_{j=1}^{n} \int_{-\infty}^{s} k_{i j}(s, \theta) x_{j}^{\sigma}(\theta) \Delta \theta\right] \Delta s \\
< & \lambda \int_{t}^{\infty} e_{r_{i}}(t, \sigma(s)) x_{i}(t)\left[a_{i i}(t) x_{i}(s)\right. \\
& \left.+\sum_{j=1, j \neq i}^{n} a_{i j}(s) x_{j}^{\sigma}\left(s-\tau_{i j}(s)\right)+\sum_{j=1}^{n} \int_{-\infty}^{s} k_{i j}(s, \theta) x_{j}^{\sigma}(\theta) \Delta \theta\right] \Delta s \\
= & \lambda \Phi_{i} x, \quad i=1,2, \ldots, n,
\end{aligned}
$$


which implies that

$$
\begin{aligned}
& \Phi(\lambda x)=\left(\Phi_{1}(\lambda x), \Phi_{2}(\lambda x), \ldots, \Phi_{n}(\lambda x)\right)^{T}<\lambda\left(\Phi_{1} x, \Phi_{2} x, \ldots, \Phi_{n} x\right)^{T}=\lambda \Phi x \\
& \quad \Rightarrow \quad \Phi(\lambda x) \ll \lambda \Phi x .
\end{aligned}
$$

Therefore, $\Phi$ is strongly superlinear. By Lemma 2.12 , system (1.4) has at most one positive almost periodic solution. As a whole, system (1.4) has a unique positive almost periodic solution. This completes the proof.

Remark 3.1 When $k_{i j} \equiv 0(i, j=1,2, \ldots, n)$ in system (1.4) with $\mathbb{T}=\mathbb{R}$, then Theorem 1.1 is obtained. So the work of this paper improves and extends the main result in [4].

From Theorem 3.1, we can easily obtain the following.

Theorem 3.2 Assume that $\left(\mathrm{H}_{1}\right)$ holds. Suppose further that all the coefficients of system (1.4) are nonnegative $\omega$-periodic functions, then system (1.4) has a unique positive $\omega$-periodic solution.

\section{Global attractivity}

In this section, we will construct suitable Lyapunov functional to study the global attractivity of system (1.4).

Now, we consider system (1.1) on time scales:

$$
x_{i}^{\Delta}(t)=x_{i}(t)\left[r_{i}(t)-a_{i i}(t) x_{i}(t)-\sum_{j=1, j \neq i}^{n} a_{i j}(t) x_{j}^{\sigma}(t)\right], \quad i=1,2, \ldots, n .
$$

Theorem 4.1 Assume that $\left(\mathrm{H}_{1}\right)$ and $\sum_{j=1, j \neq i}^{n} a_{i j}^{-}>0$ hold, $i=1,2, \ldots, n$, suppose further that there exists a constant $\gamma>0$ such that

$$
\min _{1 \leq i \leq n}\left[a_{t \in \mathbb{R}}\left[a_{i i}(t)-\sum_{j=1, j \neq i}^{n} a_{j i}(t)\right]>\gamma,\right.
$$

then system (4.1) has a unique positive almost periodic solution, which is globally attractive.

Proof By Theorem 3.1, system (4.1) has a unique positive almost periodic solution. It remains to prove the global attractivity of system (4.1).

There must exist a $\Delta$-differentiable function $\lambda$ defined on $\mathbb{T}^{\kappa}$ such that $\lambda(0)=0$ and $\lambda(t) \equiv 1$ for $t \in[1, \infty]_{\mathbb{T}}$. Define

$$
p_{f}(t)=\xi_{\mu(t)}^{-1}\left((\lambda(t) f(t))^{\Delta}\right)= \begin{cases}\frac{\exp \left\{\mu(t)(\lambda(t) f(t))^{\Delta}\right\}-1}{\mu(t)}, & \text { if } \mu(t)>0 ; \\ (\lambda(t) f(t))^{\Delta}, & \text { if } \mu(t)=0,\end{cases}
$$

where $t \in[1, \infty]_{\mathbb{T}}, f$ is a $\Delta$-differentiable function on $\mathbb{T}^{\kappa}$. Let $x_{i}(t)=e_{p y_{i}}(t, 0), i=1,2, \ldots, n$. Notice that

$$
\begin{aligned}
x_{i}(t) & =e_{p_{y_{i}}}(t, 0) \\
& =\exp \left\{\int_{0}^{t} \xi_{\mu(s)}\left(p_{y_{i}}(s)\right) \Delta s\right\}
\end{aligned}
$$




$$
\begin{aligned}
& =\exp \left\{\int_{0}^{t} \xi_{\mu(s)}\left(\xi_{\mu(s)}^{-1}\left(\left(\lambda(s) y_{i}(s)\right)^{\Delta}\right)\right) \Delta s\right\} \\
& =\exp \left\{\int_{0}^{t}\left(\lambda(s) y_{i}(s)\right)^{\Delta} \Delta s\right\} \\
& =\exp \left\{y_{i}(t)\right\}
\end{aligned}
$$

and $e_{p_{y_{i}}}^{\Delta}(t, 0)=p_{y_{i}}(t) e_{p_{y_{i}}}(t, 0), t \in[1, \infty]_{\mathbb{T}}, i=1,2, \ldots, n$. Then system (4.1) is transformed into

$$
p_{y_{i}}(t)=r_{i}(t)-a_{i i}(t) \exp \left\{y_{i}(t)\right\}-\sum_{j=1, j \neq i}^{n} a_{i j}(t) \exp \left\{y_{j}^{\sigma}(t)\right\}
$$

It is obvious that the global attractivity of system (4.1) is equivalent to that of system (4.4). So in the following, we shall prove that system (4.4) is global attractivity. Suppose that $y=\left(y_{1}, y_{2}, \ldots, y_{n}\right)^{T}$ and $z=\left(z_{1}, z_{2}, \ldots, z_{n}\right)^{T}$ are any positive solutions of system (4.4). So $y$ and $z$ satisfy (4.4) and

$$
p_{z_{i}}(t)=r_{i}(t)-a_{i i}(t) \exp \left\{z_{i}(t)\right\}-\sum_{j=1, j \neq i}^{n} a_{i j}(t) \exp \left\{z_{j}^{\sigma}(t)\right\},
$$

respectively, where $i=1,2, \ldots, n, t \in[1, \infty]_{\mathbb{T}}$.

In view of (4.2), using the mean value theorem of differential calculus on time scales leads to

$$
p_{y_{i}}(t)-p_{z_{i}}(t)= \begin{cases}e^{\delta_{i}(t)}\left[y_{i}(t)-z_{i}(t)\right]^{\Delta}, & \text { if } \mu(t)>0 ; \\ {\left[y_{i}(t)-z_{i}(t)\right]^{\Delta},} & \text { if } \mu(t)=0,\end{cases}
$$

where $\delta_{i}(t)$ lies between $\mu(t) y_{i}^{\Delta}(t)$ and $\mu(t) z_{i}^{\Delta}(t), t \in[1, \infty]_{\mathbb{T}}(\lambda(t) \equiv 1), i=1,2, \ldots, n$.

By the definition of almost periodic time scale $\mathbb{T}, \mu$ is bounded on $\mathbb{T}$. By Lemma 2.3 in [17], there exists a large point $t_{0} \in[1, \infty]_{\mathbb{T}}$ such that the species $x_{i}(t)$ of system (4.1) is bounded for $t \in\left[t_{0}, \infty\right]_{\mathbb{T}}$, and then $x_{i}^{\Delta}(t)$ is bounded for $t \in\left[t_{0}, \infty\right]_{\mathbb{T}}, i=1,2, \ldots, n$. In view of (4.3), $y_{i}(t)$ is bounded for $t \in\left[t_{0}, \infty\right]_{\mathbb{T}}, i=1,2, \ldots, n$. By Lemma 2.3, ones obtain from (4.3) that

$$
x_{i}^{\Delta}(t)=e^{y(c)} y_{i}^{\Delta}(t), \quad c \in[t, \sigma(t)], t \in\left[t_{0}, \infty\right]_{\mathbb{T}},
$$

which implies that $y_{i}^{\Delta}$ is bounded for $t \in\left[t_{0}, \infty\right]_{\mathbb{T}}$, similarly, $z_{i}$ and $z_{i}^{\Delta}$ is also bounded for $t \in\left[t_{0}, \infty\right]_{\mathbb{T}}, i=1,2, \ldots, n$.

By the boundedness of $\mu, y_{i}^{\Delta}, z_{i}^{\Delta}$, the mean value theorem of differential calculus on time scales and the definition of $\delta_{i}$, there are two positive constants $m$ and $M$ such that

$$
\begin{aligned}
& \frac{1}{M}\left|e^{y_{i}(t)}-e^{z_{i}(t)}\right| \leq\left|y_{i}(t)-z_{i}(t)\right| \leq \frac{1}{m}\left|e^{y_{i}(t)}-e^{z_{i}(t)}\right|, \\
& m<e^{-\delta_{i}(t)}<M, \quad \forall t \in\left[t_{0}, \infty\right]_{\mathbb{T}}, i=1,2, \ldots, n .
\end{aligned}
$$

Define a Lyapunov functional as follows:

$$
V(t)=\left\{\begin{array}{ll}
e_{-\eta}\left(t, t_{0}\right) \sum_{i=1}^{n}\left|y_{i}(t)-z_{i}(t)\right|, & \text { if } \mu(t)>0 ; \\
\sum_{i=1}^{n}\left|y_{i}(t)-z_{i}(t)\right|, & \text { if } \mu(t)=0
\end{array}\right\} \quad \text { for } t \in\left[t_{0}, \infty\right]_{\mathbb{T}}
$$


where $\eta$ is a positive constant. Obviously, there must exist a constant $N>0$ such that $V(t)<N$ for $t \in\left[t_{0}, \infty\right]_{\mathbb{T}}$.

Next, we will present two cases to prove the global attractivity of system (4.1).

Case I. If $\mu(t)>0$, set $\eta>\max \{n M A, \gamma\}$ and $1-\eta \mu(t)<0$, where $A=\max _{1 \leq i, j \leq n} a_{i j}^{+}, t \in$ $\left[t_{0}, \infty\right]_{\mathbb{T}}$. It follows from (4.4)-(4.6) that

$$
\begin{aligned}
{\left[y_{i}(t)-z_{i}(t)\right]^{\Delta} } & =e^{-\delta_{i}(t)}\left[p_{y_{i}}(t)-p_{z_{i}}(t)\right] \\
& =e^{-\delta_{i}(t)}\left(-a_{i i}(t)\left[e^{y_{i}(t)}-e^{z_{i}(t)}\right]+\sum_{j=1, j \neq i}^{n} a_{i j}(t)\left[e^{y_{j}^{\sigma}(t)}-e^{z_{j}^{\sigma}(t)}\right]\right) \\
& \leq e^{-\delta_{i}(t)}\left(-a_{i i}(t)\left[e^{y_{i}(t)}-e^{z_{i}(t)}\right]+\sum_{j=1, j \neq i}^{n} a_{i j}(t)\left|e^{y_{j}^{\sigma}(t)}-e^{z_{j}^{\sigma}(t)}\right|\right),
\end{aligned}
$$

where $t \in\left[t_{0}, \infty\right]_{\mathbb{T}}, i=1,2, \ldots, n$.

Calculating the upper right derivatives of $V$ along the solution of system (4.4), it follows from (4.7) that

$$
\begin{aligned}
D^{+} V(t)= & e_{-\eta}\left(t, t_{0}\right) \sum_{i=1}^{n} \operatorname{sgn}\left[y_{i}(t)-z_{i}(t)\right]\left[y_{i}^{\Delta}(t)-z_{i}^{\Delta}(t)\right] \\
& -\eta e_{-\eta}\left(t, t_{0}\right) \sum_{i=1}^{n}\left|y_{i}^{\sigma}(t)-z_{i}^{\sigma}(t)\right| \\
\leq & e_{-\eta}\left(t, t_{0}\right) \sum_{i=1}^{n} e^{-\delta_{i}(t)}\left[-a_{i i}(t)\left|e^{y_{i}(t)}-e^{z_{i}(t)}\right|+\sum_{j=1, j \neq i}^{n} a_{i j}(t)\left|e^{y_{j}^{\sigma}(t)}-e^{z_{j}^{\sigma}(t)}\right|\right] \\
& -\eta e_{-\eta}\left(t, t_{0}\right)\left|y_{i}^{\sigma}(t)-z_{i}^{\sigma}(t)\right|-\eta e_{-\eta}\left(t, t_{0}\right) \sum_{j=1, j \neq i}^{n}\left|y_{j}^{\sigma}(t)-z_{j}^{\sigma}(t)\right| \\
\leq & -m e_{-\eta}\left(t, t_{0}\right) \sum_{i=1}^{n} a_{i i}(t)\left|e^{y_{i}(t)}-e^{z_{i}(t)}\right| \\
& -(\eta-M A n) e_{-\eta}\left(t, t_{0}\right) \sum_{j=1, j \neq i}^{n}\left|y_{j}^{\sigma}(t)-z_{j}^{\sigma}(t)\right| \\
\leq & -m e_{-\eta}\left(t, t_{0}\right) \sum_{i=1}^{n} a_{i i}(t)\left|y_{i}(t)-z_{i}(t)\right| \\
\leq & -\gamma V(t),
\end{aligned}
$$

which implies from the comparison theorem that

$$
V(t) \leq e_{-\gamma}\left(t, t_{0}\right) V\left(t_{0}\right)<N e_{-\gamma}\left(t, t_{0}\right),
$$

that is,

$$
e_{-\eta}\left(t, t_{0}\right) \sum_{i=1}^{n}\left|y_{i}(t)-z_{i}(t)\right| \leq e_{-\gamma}\left(t, t_{0}\right) V\left(t_{0}\right)<N e_{-\gamma}\left(t, t_{0}\right)
$$


then

$$
\sum_{i=1}^{n}\left|y_{i}(t)-z_{i}(t)\right| \leq e_{(-\gamma) \Theta(-\eta)}\left(t, t_{0}\right) V\left(t_{0}\right)<N e_{(-\gamma) \Theta(-\eta))}\left(t, t_{0}\right), \quad t \in\left[t_{0}, \infty\right]_{\mathbb{T}} .
$$

Since $1-\eta \mu(t)<0$ and $\eta>\gamma$, then $(-\gamma) \ominus(-\eta)<0$. So it follows from (4.8) that

$$
\lim _{t \rightarrow \infty}\left|y_{i}(t)-z_{i}(t)\right|=0, \quad i=1,2, \ldots, n .
$$

Case II. If $\mu(t)=0$, then $\sigma(t)=t$ for $t \in\left[t_{0}, \infty\right]_{\mathbb{T}}$. It follows from (4.4)-(4.6) that

$$
\left[y_{i}(t)-z_{i}(t)\right]^{\Delta}=\left[p_{y_{i}}(t)-p_{z_{i}}(t)\right] \leq-a_{i i}(t)\left[e^{y_{i}(t)}-e^{z_{i}(t)}\right]+\sum_{j=1, j \neq i}^{n} a_{i j}(t)\left|e^{y_{j}(t)}-e^{z_{j}(t)}\right|,
$$

where $t \in\left[t_{0}, \infty\right]_{\mathbb{T}}, i=1,2, \ldots, n$.

Calculating the upper right derivatives of $V$ along the solution of system (4.4), it follows from (4.7) that

$$
\begin{aligned}
D^{+} V(t) & \leq \sum_{i=1}^{n}\left[-a_{i i}(t)\left|e^{y_{i}(t)}-e^{z_{i}(t)}\right|+\sum_{j=1, j \neq i}^{n} a_{i j}(t)\left|e^{y_{j}(t)}-e^{z_{j}(t)}\right|\right] \\
& =-\sum_{i=1}^{n}\left[a_{i i}(t)-\sum_{j=1, j \neq i}^{n} a_{j i}(t)\right]\left|e^{y_{i}(t)}-e^{z_{i}(t)}\right| \\
& \leq-m \gamma \sum_{i=1}^{n}\left|y_{i}(t)-z_{i}(t)\right|, \quad t \in\left[t_{0}, \infty\right]_{\mathbb{T}} .
\end{aligned}
$$

Therefore, $V$ is non-increasing. Integrating (4.9) from $t_{0}$ to $+\infty$ leads to

$$
\lim _{t \rightarrow+\infty} V(t)+m \gamma \sum_{i=1}^{n} \int_{t_{0}}^{+\infty}\left|y_{i}(s)-z_{i}(s)\right| \Delta s \leq V\left(t_{0}\right)<+\infty
$$

So

$$
\sum_{i=1}^{n} \int_{t_{0}}^{\infty}\left|y_{i}(s)-z_{i}(s)\right| \Delta s<+\infty
$$

which implies that

$$
\sum_{i=1}^{n} \lim _{s \rightarrow+\infty}\left|y_{i}(s)-z_{i}(s)\right|=0
$$

that is,

$$
\lim _{s \rightarrow+\infty}\left|y_{i}(s)-z_{i}(s)\right|=0, \quad i=1,2, \ldots, n .
$$

Thus, system (4.1) is globally attractive. This completes the proof.

When $\mathbb{T}=\mathbb{R}$, system (1.4) changes to system (1.2). Then we have: 
Theorem 4.2 Assume that $\left(\mathrm{H}_{1}\right)$ holds, suppose further that $\left(\tau_{i j}^{\prime}\right)^{+}:=\sup _{t \in \mathbb{R}} \tau_{i j}^{\prime}(t)<1$ and there exists a constant $\rho>0$ such that

$$
\min _{1 \leq i \leq n} \inf _{t \in \mathbb{R}}\left[a_{i i}(t)-\sum_{j=1, j \neq i}^{n} \frac{a_{j i}\left(v_{j i}^{-1}(t)\right)}{1-\tau_{j i}^{\prime}\left(v_{j i}^{-1}(t)\right)}-\sum_{j=1}^{n} \int_{0}^{\infty} k_{j i}(t+\theta, t) \mathrm{d} \theta\right]>\rho,
$$

where $v_{j i}^{-1}(t)$ is the inverse function of $t-\tau_{i j}(t)$. Then system (1.2) has a unique positive almost periodic solution, which is globally attractive.

Proof By Theorem 3.1, system (1.2) has a unique positive almost periodic solution. It remains to prove the global attractivity of system (1.2).

Suppose that $x=\left(x_{1}, x_{2}, \ldots, x_{n}\right)^{T}$ and $y=\left(y_{1}, y_{2}, \ldots, y_{n}\right)^{T}$ are any positive solutions of system (1.2).

Define a Lyapunov functional as follows:

$$
V(t)=\sum_{i=1}^{n}\left[V_{i 1}(t)+V_{i 2}(t)+V_{i 3}(t)\right]
$$

where

$$
\begin{aligned}
& V_{i 1}(t)=\left|\ln x_{i}(t)-\ln y_{i}(t)\right|, \\
& V_{i 2}(t)=\sum_{j=1, j \neq i}^{n} \int_{t-\tau_{i j}(t)}^{t} \frac{a_{i j}\left(v_{i j}^{-1}(s)\right)}{1-\tau_{i j}^{\prime}\left(v_{i j}^{-1}(s)\right)}\left|x_{j}(s)-y_{j}(s)\right| \mathrm{d} s, \\
& V_{i 3}(t)=\sum_{j=1}^{n} \int_{0}^{\infty} \int_{t-\theta}^{t} k_{i j}(s+\theta, s)\left|x_{j}(s)-y_{j}(s)\right| \mathrm{d} s \mathrm{~d} \theta, \quad t \in \mathbb{R}, i=1,2, \ldots, n .
\end{aligned}
$$

Calculating the upper right derivatives of $V$ along the solution of system (1.2), it follows that

$$
\begin{aligned}
D^{+} V_{i 1}(t)= & \operatorname{sgn}\left[x_{i}(t)-y_{i}(t)\right]\left[\frac{x_{i}^{\prime}(t)}{x_{i}(t)}-\frac{y_{i}^{\prime}(t)}{y_{i}(t)}\right] \\
\leq & -a_{i i}(t)\left|x_{i}(t)-y_{i}(t)\right|+\sum_{j=1, j \neq i}^{n} a_{i j}(t)\left|x_{j}\left(t-\tau_{i j}(t)\right)-y_{j}\left(t-\tau_{i j}(t)\right)\right| \\
& +\sum_{j=1}^{n} \int_{-\infty}^{t} k_{i j}(t, \theta)\left|x_{j}(\theta)-y_{j}(\theta)\right| \mathrm{d} \theta, \\
D^{+} V_{i 2}(t) \leq & \sum_{j=1, j \neq i}^{n} \frac{a_{i j}\left(v_{i j}^{-1}(t)\right)}{1-\tau_{i j}^{\prime}\left(v_{i j}^{-1}(t)\right)}\left|x_{j}(t)-y_{j}(t)\right| \\
& -\sum_{j=1, j \neq i}^{n} a_{i j}(t)\left|x_{j}\left(t-\tau_{i j}(t)\right)-y_{j}\left(t-\tau_{i j}(t)\right)\right|, \\
D^{+} V_{i 3}(t)= & \sum_{j=1}^{n} \int_{0}^{\infty} k_{i j}(t+\theta, t)\left|x_{j}(t)-y_{j}(t)\right| \mathrm{d} \theta \\
& -\sum_{j=1}^{n} \int_{-\infty}^{t} k_{i j}(t, \theta)\left|x_{j}(\theta)-y_{j}(\theta)\right| \mathrm{d} \theta, \quad t \in \mathbb{R}, i=1,2, \ldots, n .
\end{aligned}
$$


Together with (4.10)-(4.12), we get

$$
\begin{aligned}
D^{+} V(t) & =\sum_{i=1}^{n}\left[D^{+} V_{i 1}(t)+D^{+} V_{i 2}(t)+D^{+} V_{i 3}(t)\right] \\
& \leq-\sum_{i=1}^{n}\left[a_{i i}(t)-\sum_{j=1, j \neq i}^{n} \frac{a_{j i}\left(v_{j i}^{-1}(t)\right)}{1-\tau_{j i}^{\prime}\left(v_{j i}^{-1}(t)\right)}-\sum_{j=1}^{n} \int_{0}^{\infty} k_{j i}(t+\theta, t) \mathrm{d} \theta\right]\left|x_{i}(t)-y_{i}(t)\right| \\
& \leq-\rho \sum_{i=1}^{n}\left|x_{i}(t)-y_{i}(t)\right| .
\end{aligned}
$$

Similar to the argument as that in Theorem 4.1, one has

$$
\lim _{s \rightarrow+\infty}\left|x_{i}(s)-y_{i}(s)\right|=0, \quad i=1,2, \ldots, n
$$

Thus, system (1.2) is globally attractive. This completes the proof.

Remark 4.1 From Theorem 4.2, system (1.2) admits a unique globally attractive positive almost periodic solution without condition $\left(\mathrm{T}_{2}\right)$ of Theorem 1.2. Therefore, the main result in this paper improves and extends the work in $[5,6]$.

From Theorems 4.1 and 4.2, we can easily obtain the following.

Theorem 4.3 Assume that all conditions of Theorem 4.1 hold. Suppose further that all the coefficients of system (4.1) are nonnegative $\omega$-periodic functions, then system (4.1) has a unique positive $\omega$-periodic solution, which is globally attractive.

Corollary 4.1 Assume that all conditions of Theorem 4.2 hold. Suppose further that all the coefficients of system (1.2) are nonnegative $\omega$-periodic functions, then system (1.2) has a unique positive $\omega$-periodic solution, which is globally attractive.

\section{Applications of main theorems to global attractive almost periodic solutions}

Example 5.1 Consider the following two species Lotka-Volterra system:

$$
\left\{\begin{array}{l}
x^{\Delta}(t)=x(t)\left[1-a_{1}(t) x(t)-a_{12}(t) y^{\sigma}(t)\right] \\
y^{\Delta}(t)=y(t)\left[1-a_{2}(t) y(t)-a_{21}(t) x^{\sigma}(t)\right]
\end{array}\right.
$$

where

$$
\left(\begin{array}{l}
a_{1}(t) \\
a_{2}(t)
\end{array}\right)=\left(\begin{array}{c}
2+0.01 \sin (\sqrt{2} t) \\
3+0.01 \cos (\sqrt{13} t)
\end{array}\right), \quad\left(\begin{array}{l}
a_{12}(t) \\
a_{21}(t)
\end{array}\right)=\left(\begin{array}{c}
6+0.01|\sin (\sqrt{7} t)| \\
6+0.01|\sin (t)|
\end{array}\right),
$$

$\forall t \in \mathbb{T}=\{n q: n \in \mathbb{Z}\}, q>0$. Then system (5.1) has a unique positive almost periodic solution.

Proof Obviously, $r_{1}^{-}=r_{2}^{-}=1>0$ and $a_{12}^{-}=a_{21}^{-}=6$, which imply that $\left(\mathrm{H}_{1}\right)$ of Theorem 3.1 holds. By Theorem 3.1, system (5.1) has a unique positive almost periodic solution. 
Remark 5.1 When $\mathbb{T}=\mathbb{R}$, it is impossible to obtain the existence of positive almost periodic solution of system (5.1) by Theorem 1.1 in Section 1.

Proof In view of Theorem 1.1 in Section 1 and system (5.1), one has

$$
G_{1}(t)=\left[\int_{-\infty}^{t} e^{-(t-s)} a_{1}(s) \mathrm{d} s\right]^{-1} \geq \frac{1}{3}
$$

which implies that

$$
m\left[r_{1}(t)-\sum_{j=1, j \neq i}^{2} a_{1 j}(t) G_{1}(t)\right] \leq 1-\frac{1}{3} \times 6=-1<0 .
$$

So $\left(\mathrm{N}_{2}\right)$ of Theorem 1.1 is invalid. This completes the proof.

Example 5.2 Consider the following two species Lotka-Volterra system:

$$
\left\{\begin{aligned}
x^{\Delta}(t)= & x(t)\left[10^{-3}-a_{1}(t) x(t)-a_{12}(t) y^{\sigma}\left(t-0.1 \sin ^{2}(\sqrt{2} t)\right)\right. \\
& \left.-\int_{-\infty}^{t} e^{-t+\theta} y^{\sigma}(\theta) \Delta \theta\right], \\
y^{\Delta}(t)= & y(t)\left[1-a_{2}(t) y(t)-a_{21}(t) x^{\sigma}\left(t-0.1 \cos ^{2}(\sqrt{3} t)\right)\right. \\
& \left.-\int_{-\infty}^{t} e^{-2 t+2 \theta} x^{\sigma}(\theta) \Delta \theta\right],
\end{aligned}\right.
$$

where

$$
\left(\begin{array}{l}
a_{1}(t) \\
a_{2}(t)
\end{array}\right)=\left(\begin{array}{c}
2+0.01 \sin (\sqrt{2} t) \\
3+0.01 \cos (\sqrt{13} t)
\end{array}\right), \quad\left(\begin{array}{l}
a_{12}(t) \\
a_{21}(t)
\end{array}\right)=\left(\begin{array}{c}
0.01+0.01|\sin (\sqrt{7} t)| \\
0.01+0.01|\sin (t)|
\end{array}\right), \quad \forall t \in \mathbb{T} .
$$

Then one finds that system (5.2) has a unique positive almost periodic solution. If $\mathbb{T}=\mathbb{R}$, the almost periodic solution of system (5.2) is even globally attractive.

Proof Similar to the argument in Example 5.1, all of the conditions in Theorem 3.1 are satisfied. By Theorem 3.1, system (5.2) has a unique positive almost periodic solution.

If $\mathbb{T}=\mathbb{R}$, by an easy calculation,

$$
\left(\tau_{12}^{\prime}\right)^{+}=\left(\tau_{21}^{\prime}\right)^{+}=0.14, \quad \int_{0}^{\infty} k_{12}(t+\theta, t) \mathrm{d} \theta=\int_{0}^{\infty} k_{21}(t+\theta, t) \mathrm{d} \theta=1,
$$

which imply that all of the conditions in Theorem 4.2 hold. By Theorem 4.2, system (5.2) has a unique positive almost periodic solution, which is globally attractive. This completes the proof.

Remark 5.2 When $\mathbb{T}=\mathbb{R}$, it is impossible to obtain the existence, uniqueness and global attractivity of positive almost periodic solution of system (5.2) by Theorem 1.2 in Section 1.

Proof In view of Theorem 1.2 in Section 1 and system (5.2), one has

$$
M_{2}=\sup _{t \in \mathbb{R}} \frac{r_{2}(t)}{a_{2}(t)} \geq \frac{1}{4}
$$


which implies that

$$
\inf _{t \in \mathbb{R}}\left[r_{1}(t)-a_{12}(t) M_{2}\right] \leq 0.001-0.01 \times \frac{1}{4}<0 .
$$

So $\left(\mathrm{T}_{2}\right)$ of Theorem 1.2 is invalid. This completes the proof.

\section{Conclusion}

In this paper, some sufficient conditions are established for the existence, uniqueness and global attractivity of positive almost periodic solution for a delayed Lotka-Volterra system on time scales by using the theory of exponential dichotomy on time scales, fixed point theory based on monotone operator and Lyapunov functional. The main results obtained in this paper are completely new even in case of the time scale $\mathbb{T}=\mathbb{R}$ or $\mathbb{Z}$. The work of this paper improves the previously known results. It is important to notice that the approach used in this paper can be extended to other types of biological models of first order $[8,9,19$, 20]. Future work will include biological dynamic systems based on impulsive differential equations $[25,26]$.

\section{Competing interests}

The authors declare that they have no competing interests.

\section{Authors' contributions}

$Y L$ participated in the design of the study and drafted the manuscript. LX read and approved the final version of the manuscript.

\section{Acknowledgements}

The authors thank the reviewers for their constructive remarks that led to the improvement of the original manuscript.

Received: 17 September 2013 Accepted: 3 March 2014 Published: 25 Mar 2014

\section{References}

1. Gao, P: Hamiltonian structure and first integrals for the Lotka-Volterra systems. Phys. Lett. A 273, 85-96 (2000)

2. Provata, A, Tsekouras, GA: Spontaneous formation of dynamical patterns with fractal fronts in the cyclic lattice Lotka-Volterra model. Phys. Rev. E 67, Article ID 056602 (2003)

3. Geisshirt, K, Praestgaard, E, Toxvaerd, S: Oscillating chemical reactions and phase separation simulated by molecular dynamics. J. Chem. Phys. 107, 9406-9412 (1997)

4. $\mathrm{Ni}, \mathrm{H}$, Tian, LX, Yao, HX: On the positive almost periodic solutions of a class of nonlinear Lotka-Volterra type system with feedback control. J. Appl. Math. 2012, Article ID 135075 (2012)

5. Teng, ZD: On the positive almost periodic solutions of a class of Lotka-Volterra type systems with delays. J. Math. Anal. Appl. 249, 433-444 (2000)

6. Wang, Q, Dai, BX: Almost periodic solution for $n$-species Lotka-Volterra competitive system with delay and feedback controls. Appl. Math. Comput. 200, 133-146 (2008)

7. Li, Z, Chen, FD, He, MX: Almost periodic solutions of a discrete Lotka-Volterra competition system with delays. Nonlinear Anal., Real World Appl. 12, 2344-2355 (2011)

8. Zhang, TW, Gan, XR: Existence and permanence of almost periodic solutions for Leslie-Gower predator-prey model with variable delays. Electron. J. Differ. Equ. 2013, 105 (2013)

9. Zhang, TW, Gan, XR: Almost periodic solutions for a discrete fishing model with feedback control and time delays. Commun. Nonlinear Sci. Numer. Simul. 19, 150-163 (2014)

10. Hilger, S: Ein Maßkettenkalkül mit Anwendung auf Zentrumsmannigfaltigkeiten. PhD thesis, Universität Würzburg (1988)

11. Bohner, M, Peterson, A: Dynamic Equations on Time Scales: An Introduction with Applications. Birkhäuser, Boston (2001)

12. Kaufmann, ER, Raffoul, YN: Periodic solutions for a neutral nonlinear dynamical equation on a time scale. J. Math. Anal. Appl. 319, 315-325 (2006)

13. Li, YK, Wang, C: Uniformly almost periodic functions and almost periodic solutions to dynamic equations on time scales. Abstr. Appl. Anal. 2011, Article ID 341520 (2011)

14. Zhang, J, Fan, M, Zhu, H: Existence and roughness of exponential dichotomies of linear dynamic equations on time scales. Comput. Math. Appl. 59, 2658-2675 (2010)

15. Li, YK, Wang, C: Almost periodic functions on time scales and applications. Discrete Dyn. Nat. Soc. 2011, Article ID 727068 (2011)

16. Li, YK, Yang, L: Almost periodic solutions for neutral-type BAM neural networks with delays on time scales. J. Appl. Math. 2013, Article ID 942309 (2013) 
17. Hu, M, Wang, LL: Unique existence theorem of solution of almost periodic differential equations on time scales. Discrete Dyn. Nat. Soc. 2012, Article ID 240735 (2012)

18. Grace, SR, Agarwal, RP, Bohner, M, O'Regan, D: Oscillation of second-order strongly superlinear and strongly sublinear dynamic equations. Commun. Nonlinear Sci. Numer. Simul. 14, 3463-3471 (2009)

19. Shu, JY, Zhang, TW: Multiplicity of almost periodic oscillations in a harvesting mutualism model with time delays. Dyn. Contin. Discrete Impuls. Syst., Ser. B 20, 463-483 (2013)

20. Zhang, TW: Multiplicity of positive almost periodic solutions in a delayed Hassell-Varley-type predator-prey model with harvesting on prey. Math. Methods Appl. Sci. (2013). doi:10.1002/mma.2826

21. Kostrykin, V, Oleynik, A: An intermediate value theorem for monotone operators in ordered Banach spaces. Fixed Point Theory Appl. 2012, 211 (2012)

22. Guo, DJ: Nonlinear Functional Analysis. Shandong Science and Technology Press, Jinan (2003) (in Chinese)

23. Agarwal, RP, Bohner, M, Rehak, P: Half-linear dynamic equations. In: Nonlinear Analysis and Applications: To V. Lakshmikantham on His 80th Birthday, vol. 1, pp. 1-57. Kluwer Academic, Dordrecht (2003)

24. Aulbach, B, Neidhart, L: Integration on measure chains. In: Proceedings of the Sixth International Conference on Difference Equations, pp. 239-252. CRC Press, Boca Raton (2004)

25. Zhang, TW, Li, YK: Positive periodic solutions for a generalized impulsive $n$-species Gilpin-Ayala competition system with continuously distributed delays on time scales. Int. J. Biomath. 4, 23-34 (2011)

26. Zhang, TW, Li, YK, Ye, Y: On the existence and stability of a unique almost periodic solution of Schoener's competition model with pure-delays and impulsive effects. Commun. Nonlinear Sci. Numer. Simul. 17, 1408-1422 (2012)

10.1186/1687-1847-2014-96

Cite this article as: Liao and Xu: Almost periodic solution for a delayed Lotka-Volterra system on time scales. Advances in Difference Equations 2014, 2014:96

\section{Submit your manuscript to a SpringerOpen ${ }^{\circ}$ journal and benefit from:}

- Convenient online submission

- Rigorous peer review

- Immediate publication on acceptance

- Open access: articles freely available online

- High visibility within the field

- Retaining the copyright to your article 